\title{
A time domain random flicker band-limited Gaussian noise jamming algorithm for LMS- based GPS
}

\author{
Tao $\mathrm{Wu}^{*}$, Jixiang $\mathrm{Wu}$, Hui Wang, Ligang Cui, and Yinsong Yang \\ Air Force Communication NCO Academy, China
}

\begin{abstract}
Global position systems (GPS) receiver based on least mean square (LMS) could resist the interference by null jamming direction. Considering that the existing band-limited Gaussian noise jamming signals were easily suppressed by LMS-based GPS receivers, a time domain random flicker band-limited Gaussian noise jamming algorithm was proposed to improve its performance. By disturbing the convergence of LMS, it could achieve the purpose of suppressing the LMS-based GPS. Simulation result shows that the proposed algorithm has an average performance gain of $2.7 \mathrm{~dB} \sim 4.6 \mathrm{~dB}$ under different number of interferences compared with band-limited Gaussian noise.
\end{abstract}

\section{Introduction}

GPS jamming is mainly divided into deceptive jamming and suppressing jamming. It is very difficult to implement deceptive jamming because the long length of $\mathrm{P}(\mathrm{Y})$ code in GPS spread spectrum code and encryption processing. Blanket jamming can transmit jamming signals with higher power than GPS signal, which increases the difficulty of GPS receiver acquisition and tracking, and makes GPS receiver unable to decode correctly. By using adaptive algorithm to adjust the weight of the antenna array, the nulling GPS can suppress the interference and achieve better anti-jamming effect.

Several jamming algorithms have been proposed for GPS ${ }^{[1-5]}$. The BER of receiver under different jamming modes is studied in [1] and presents a new broadband comb spectrum jamming type. In [2], The influence of pulse and Gaussian white noise on C/A code and $\mathrm{M}$ code is studied and proposed a method of judging the optimal jamming signal by using error rate. The optimal parameter selection strategy of Gaussian white noise interference is studied in [3]. Reference [4] show that the smaller LMS step size can decrease the mis-adjustment. Reference [5] analyses the influence of continuous wave (CW) interference on the acquisition performance of GPS receivers.

Previous interference methods do not focus on the LMS-based GPS receivers. In reference [6], the effect of band-limited Gaussian noise interference with different bandwidths is assessed. In reference [7], a pulse jamming algorithm is proposed for nulling GPS. The relative delay between multiple interferences is adjusted periodically, and a

\footnotetext{
* Corresponding author: wutao996@126.com
} 
certain performance gain is obtained. Reference [8] show the jamming optimal power difference for LMS-based GPS receivers.

In this paper, we propose a novel jamming method for LMS-based GPS receiver. Firstly, the LMS-based GPS is introduced. And then, the proposed jamming algorithm is developed in detail. Under the same jamming power, the smaller null depth of the pattern in the interference signal pattern indicates that the stronger the disturbance ability of the jamming signal to the adaptive algorithm, the better the jamming effect. Therefore, the null depth of the pattern can be used as an index to describe the performance of jamming signals. The proposed jamming algorithm can obtain better performance on null depth of pattern compared with the band-limited Gaussian noise.

The rest of this paper is organized as follows. Section 2 describes the system model, including interference signal model, antenna array, LMS algorithm and null depth of pattern. In Section 3, a time domain random flicker band-limited Gaussian noise jamming algorithm is proposed. The algorithm uses band-limited Gaussian noise as jamming signal, flickers in time domain, and pulse duration and interval time are random in a certain range. In Section 4, the null depth of this algorithm and ordinary band-limited Gaussian noise interference is verified by experimental simulation. Section 5 provides a concluding remark to summarize this paper.

\section{System model}

The pattern of LMS-based GPS receiver model is shown in Figure 1. After the antenna array receives the interference signal, the weight $W$ of each antenna element is calculated by the adaptive algorithm based on LMS. The signal weighted by the weight $W$ has the function of nulling the interference direction signal. Through the weight $W$, the pattern $G(\theta, \varphi)$ can be calculated (where $\theta$ is the elevation angle and $\varphi$ is the azimuth angle). Through the pattern, we can know the null depth of the antenna array and the adaptive algorithm for each direction. Next, the receiving antenna array signal model, adaptive algorithm based on LMS and null depth of pattern are described respectively.

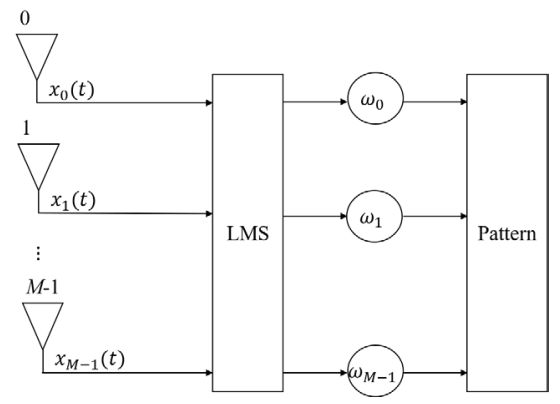

Fig. 1. The pattern of LMS-based GPS receiver model.

\subsection{Receiving antenna array signal model}

The reference array element is selected so that when the transmitted signal reaches the reference element, the received signal is

$$
x_{0}(t)=A(t) e^{j(\omega t+\varphi)}
$$

where $A(t)$ is the signal amplitude, $\omega$ is angular frequency and $\varphi$ is the initial phase of the signal. Assuming that there are $M$ elements in the antenna array. The delay between the i-th $(\mathrm{i}=1,2, \ldots, M)$ element and the reference element is $\tau_{i}$. Then The received signal of the $\mathrm{i}$-th element can be expressed as: 


$$
x_{i}(t)=x_{0}\left(t-\tau_{i}\right)=A\left(t-\tau_{i}\right) e^{j\left[\omega\left(t-\tau_{i}\right)+\varphi\right]}=A\left(t-\tau_{i}\right) e^{j(\omega t+\varphi)} e^{-j \omega \tau_{i}}
$$

for narrow-band signals we have $A\left(t-\tau_{i}\right) \approx A(t)$, so we get the expression as follows:

$$
x_{i}(t) \approx A(t) e^{j(\omega t+\varphi)} e^{-j \omega \tau_{i}}=x_{0}(t) e^{-j \omega \tau_{i}}
$$

write $x_{i}(t)$ in matrix form $X$, we get the expression as follows:

where

$$
X=A x_{0}
$$

$$
\begin{gathered}
X=\left[\begin{array}{lllll}
x_{0} & x_{1} & x_{2} & \ldots & x_{M-1}
\end{array}\right]^{T} \\
A=\left[\begin{array}{lllll}
1 & e^{-j \omega \tau_{1}} & e^{-j \omega \tau_{2}} & \ldots & e^{-j \omega \tau_{M-1}}
\end{array}\right]^{T}
\end{gathered}
$$

where $A$ is called the guidance vector.

Assuming that the received signal consists of GPS signal, jamming signal and additive Gaussian white noise, the received signal $\mathrm{R}$ of array element is expressed as follows:

$$
R=\sum_{i=1}^{N_{s}} A_{S_{i}} S_{i}+\sum_{j=1}^{N_{J}} A_{j} J_{j}+N
$$

where $A_{S_{i}}$ refers to the guidance vector of the i-th GPS signal, $A_{j}$ is the guidance vector of the j-th jamming signal, $S_{i}$ represents the received signal of the $\mathrm{i}$-th GPS signal at the reference array element, $J_{j}$ is the received signal of the $\mathrm{j}$-th jamming signal at the reference array element, and $N$ is the additive Gaussian white noise. $N_{S}$ is the number of GPS signals. $N_{J}$ is the number of jamming signals.

The antenna array generally includes uniform linear array, planar array and uniform circular array. The uniform circular array is more suitable for GPS receiver because of its better directional characteristics. Figure 2 is a uniform circular array with seven elements. For M-element antenna array, its degree of freedom is $\mathrm{M}-1$, so the jamming signal in $\mathrm{M}-1$ direction can be nullified at most.

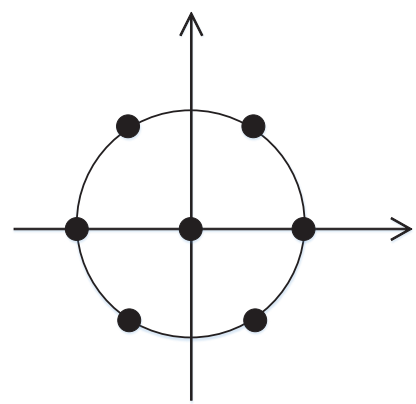

Fig. 2. Uniform circular array with seven elements.

For a uniform circular array with seven elements, it is easy to know that its guidance vector is

$$
A(\theta, \varphi)=\left[\begin{array}{c}
1 \\
e^{-j \frac{2 \pi}{\lambda} r \sin \theta \cos \left(\varphi-\varphi_{1}\right)} \\
e^{-j \frac{2 \pi}{\lambda} r \sin \theta \cos \left(\varphi-\varphi_{2}\right)} \\
e^{-j \frac{2 \pi}{\lambda} r \sin \theta \cos \left(\varphi-\varphi_{3}\right)} \\
e^{-j \frac{2 \pi}{\lambda} r \sin \theta \cos \left(\varphi-\varphi_{4}\right)} \\
e^{-j \frac{2 \pi}{\lambda} r \sin \theta \cos \left(\varphi-\varphi_{5}\right)} \\
e^{-j \frac{2 \pi}{\lambda} r \sin \theta \cos \left(\varphi-\varphi_{6}\right)}
\end{array}\right]
$$

where $\lambda$ is the signal wavelength, $r$ is the distance between the antenna array element and the reference array element, $\theta$ is the signal elevation angle, $\varphi$ is the signal azimuth angle, $\left[\varphi_{1} \varphi_{2} \varphi_{3} \varphi_{4} \varphi_{5} \varphi_{6}\right]=\left[0 \frac{\pi}{3} \frac{2 \pi}{3} \pi \frac{4 \pi}{3} \frac{5 \pi}{3}\right]$ is the antenna array element azimuth. 


\subsection{Adaptive algorithm based on LMS}

The LMS algorithm has been widely used in the GPS receivers ${ }^{[9]}$. LMS algorithm is derived from minimum mean square error (MMSE) criterion. LMS algorithm minimizes the mean square error between the filtered output signal and the expected signal. The input signal vector is assumed to be:

the weight vector is as follows:

$$
X(k)=\left[x_{1}(\mathrm{k}) x_{2}(\mathrm{k}) \ldots x_{M-1}(\mathrm{k})\right]^{T}
$$

then the output signal of the filter is:

$$
W=\left[\begin{array}{llll}
w_{1} & w_{2} & \ldots & w_{M-1}
\end{array}\right]^{T}
$$

$$
y(k)=W^{T} X(k)
$$

If the reference signal is $d(k)$, then the error signal is:

$$
e(k)=d(k)-y(k)
$$

LMS algorithm uses steepest descent method to get weight vector:

$$
W(k+1)=W(k)-\mu \nabla_{w} E\left\{|e(k)|^{2}\right\}
$$

Finally, the LMS algorithm can be written as follows:

$$
W(k+1)=W(k)-2 \mu X(k) e(k)^{T}
$$

where $\mu$ is the iteration step size, which equal to variable step. ${ }^{[10]}$ The step size should be satisfied as follows:

$$
0<\mu<\frac{1}{2 \lambda_{\max }}
$$

where $\lambda_{\max }$ is the maximum eigenvalue of $X(k)$ autocorrelation matrix.

\subsection{Null depth of pattern}

Suppose LMS algorithm obtains the optimal weight $W_{\text {opt }}=\left[\begin{array}{llll}W_{1} & W_{2} & \ldots & W_{M-1}\end{array}\right]^{T}$, then the weight of each element can be expressed as:

Then the pattern is expressed as:

$$
W=\left[1-W_{1}-W_{2} \ldots-W_{M-1}\right]^{T}
$$

$$
G(\theta, \varphi)=W^{T} A(\theta, \varphi)
$$

\section{Time domain random flicker band-limited Gaussian noise jamming algorithm}

The band-limited Gaussian noise is used as the jamming signal and randomly flickers in the time domain. The complex domain expression of interference signal is as follows:

$$
x(n T)=r(n T)\left[f_{1}(n T) \cos \left(2 \pi f_{c} n T+\beta\right)+j f_{2}(n T) \sin \left(2 \pi f_{c} n T+\beta\right)\right]
$$

where $f_{c}$ is carrier frequency, $\beta$ is initial phase, $T=1 / f_{s}$ is the sampling period. $f_{i}(n T)(i=1,2)$ denotes white Gaussian noise after $H(z)$ filtering:

$$
f_{i}(n T)=h * g_{i}(n T)
$$

where * denotes convolution. $g_{i}(n T)$ is the Gaussian white noise. $h$ denote the filter, and its Z-domain expression is:

$$
H(z)=\frac{1}{1-0.9 z^{-1}}
$$

$r(n T)$ is the random flicker function, which is expressed as follows:

$$
r(n T)=\left\{\begin{array}{cc}
1 & n \in\left(F_{a}(2 k), F_{a}(2 k+1)\right], k=0,1,2, \ldots \\
0 & \text { other }
\end{array}\right.
$$

where $F_{a}(k)=\sum_{i=1}^{k} a(k), F_{a}(0)=0$, where $a(k) \epsilon[400,600]$ is a random sequence uniformly distributed in an interval.

The time domain random flicker band-limited Gaussian noise jamming algorithm uses a certain random algorithm, and the jamming effectiveness varies greatly under different 
random conditions. In order to describe the algorithm performance more clearly, the mean value of the null depth of a large number of samples will be used to describe the algorithm performance.

\section{Simulation experiment}

In this section, we called the time domain random flicker band-limited Gaussian noise jamming algorithm as this paper algorithm. The number of interference signals $N_{J}$ is $1 \sim 6$ test samples respectively. The average value of the LMS algorithm convergence time (the number of sampling points is greater than 4000) is taken for a single test signal's null depth. In Table 1, the average value of 1000 groups of test samples is taken as the null depth. The other parameters are as follows: the antenna array is a uniform circular array with 7 elements $(\mathrm{M}=7)$; the antenna array radius is $r=\lambda / 2$; the sampling rate is $f_{s}=3.2 \mathrm{GHz}$; the number of samples is $50 \mathrm{~K}$; the number of GPS signals is $N_{s}=1$; GPS signal angle is $70^{\circ}$ and azimuth angle is $10^{\circ}$; jamming signal elevation angle is $70^{\circ}$ and azimuth angle is $40^{\circ}$, $70^{\circ}, 100^{\circ}, 150^{\circ}, 250^{\circ}$ and $320^{\circ}$ respectively; the signal-to-noise ratio $\mathrm{S} / \mathrm{N}=-30 \mathrm{~dB}$ and the jamming-to-noise ratio $\mathrm{J} / \mathrm{N}=30 \mathrm{~dB}$ (the total interference power is taken for multiple interferences).

Table 1. Comparison of null depth between band-limited Gaussian noise interference and this paper algorithm interference.

\begin{tabular}{|c|c|c|c|c|}
\hline $\begin{array}{c}\text { Interference } \\
\text { number }\end{array}$ & $\begin{array}{c}\text { Jamming } \\
\text { azimuth }\end{array}$ & $\begin{array}{c}\text { Gaussian } \\
\text { white noise }\end{array}$ & $\begin{array}{c}\text { This paper } \\
\text { algorithm }\end{array}$ & $\begin{array}{c}\text { Gain of } \\
\text { performance }\end{array}$ \\
\hline 1 & $40^{\circ}$ & $48.8494 \mathrm{~dB}$ & $46.0916 \mathrm{~dB}$ & $2.7578 \mathrm{~dB}$ \\
\hline \multirow{3}{*}{2} & $40^{\circ}$ & $48.6734 \mathrm{~dB}$ & $45.8147 \mathrm{~dB}$ & $2.8587 \mathrm{~dB}$ \\
\cline { 2 - 5 } & $70^{\circ}$ & $48.6535 \mathrm{~dB}$ & $45.7740 \mathrm{~dB}$ & $2.8795 \mathrm{~dB}$ \\
\hline \multirow{4}{*}{3} & $40^{\circ}$ & $50.0975 \mathrm{~dB}$ & $46.9006 \mathrm{~dB}$ & $3.1969 \mathrm{~dB}$ \\
\cline { 2 - 5 } & $70^{\circ}$ & $50.1576 \mathrm{~dB}$ & $47.1126 \mathrm{~dB}$ & $3.0450 \mathrm{~dB}$ \\
\cline { 2 - 5 } & $100^{\circ}$ & $50.0747 \mathrm{~dB}$ & $46.8851 \mathrm{~dB}$ & $3.1896 \mathrm{~dB}$ \\
\hline \multirow{5}{*}{5} & $40^{\circ}$ & $47.5504 \mathrm{~dB}$ & $44.1226 \mathrm{~dB}$ & $3.4278 \mathrm{~dB}$ \\
\cline { 2 - 5 } & $70^{\circ}$ & $47.6725 \mathrm{~dB}$ & $44.5964 \mathrm{~dB}$ & $3.0761 \mathrm{~dB}$ \\
\cline { 2 - 5 } & $100^{\circ}$ & $47.5858 \mathrm{~dB}$ & $44.3478 \mathrm{~dB}$ & $3.2380 \mathrm{~dB}$ \\
\hline \multirow{5}{*}{5} & $150^{\circ}$ & $47.5810 \mathrm{~dB}$ & $44.3811 \mathrm{~dB}$ & $3.1999 \mathrm{~dB}$ \\
\hline & $40^{\circ}$ & $42.0577 \mathrm{~dB}$ & $38.5590 \mathrm{~dB}$ & $3.4987 \mathrm{~dB}$ \\
\cline { 2 - 5 } & $70^{\circ}$ & $41.4837 \mathrm{~dB}$ & $37.9738 \mathrm{~dB}$ & $3.5099 \mathrm{~dB}$ \\
\cline { 2 - 5 } & $100^{\circ}$ & $41.6031 \mathrm{~dB}$ & $38.3144 \mathrm{~dB}$ & $3.2887 \mathrm{~dB}$ \\
\cline { 2 - 5 } & $150^{\circ}$ & $41.4515 \mathrm{~dB}$ & $37.2523 \mathrm{~dB}$ & $4.1992 \mathrm{~dB}$ \\
\cline { 2 - 5 } & $250^{\circ}$ & $41.3655 \mathrm{~dB}$ & $37.2227 \mathrm{~dB}$ & $4.1428 \mathrm{~dB}$ \\
\hline \multirow{5}{*}{6} & $40^{\circ}$ & $29.4193 \mathrm{~dB}$ & $24.7766 \mathrm{~dB}$ & $4.6427 \mathrm{~dB}$ \\
\cline { 2 - 5 } & $70^{\circ}$ & $30.0382 \mathrm{~dB}$ & $26.4614 \mathrm{~dB}$ & $3.5768 \mathrm{~dB}$ \\
\cline { 2 - 5 } & $100^{\circ}$ & $29.9667 \mathrm{~dB}$ & $26.3129 \mathrm{~dB}$ & $3.6538 \mathrm{~dB}$ \\
\cline { 2 - 5 } & $150^{\circ}$ & $29.8727 \mathrm{~dB}$ & $25.7182 \mathrm{~dB}$ & $4.1545 \mathrm{~dB}$ \\
\cline { 2 - 5 } & $250^{\circ}$ & $29.6845 \mathrm{~dB}$ & $25.2337 \mathrm{~dB}$ & $4.4508 \mathrm{~dB}$ \\
\cline { 2 - 5 } & $320^{\circ}$ & $29.8959 \mathrm{~dB}$ & $25.9098 \mathrm{~dB}$ & $3.9861 \mathrm{~dB}$ \\
\hline
\end{tabular}

It can be seen from Table 1 that when the interference number $N_{J}$ is $1 \sim 5$, whether this paper algorithm or the band-limited Gaussian noise algorithm, the difference of null depth is not significant, but it decreases obviously when the number of interferences reaches 6 . The relative gain between this paper algorithm and the band-limited Gaussian noise jamming algorithm increases with the increase of the number of interferences. The 
performance gain of the proposed algorithm is about $2.7 \mathrm{~dB} \sim 4.6 \mathrm{~dB}$ relative to the interference of band-limited Gaussian noise.

\section{Summary}

This paper briefly described the GPS adaptive algorithm based on LMS, and proposes a time domain random flicker band-limited Gaussian noise jamming algorithm for LMSbased GPS. The performance of the algorithm is analyzed by the interference null depth. The algorithm uses the interference signal with random duration and jamming time to disturb the convergence of LMS, so as to obtain better null depth gain. Simulation results show that the performance of the proposed algorithm is better than that of band-limited Gaussian noise under different interferences.

\section{References}

1. Mao Hu, Wu Dewei, Lu Hu, Yan Zhanjie. Analysis of a New Wideband Blanket Jamming Type to GPS Receiver [J]. Journal of Electronics \& Information Technology, 12(36), 2929-2933 (2014)

2. Wang Jiao, Su Zhong, Zhang Yuexia. Study on Optimal Jamming Signal of GPS System[J]. Computer Measurement \& Control, 24(4), 257-267 (2016)

3. Mao Hu, Wu Dewei. Analysis of Jamming Parameters Selecting of Band-limited Gaussian Noise to GPS Military Signals[J]. Journal of air force engineering university(natural science edition), 15(6), 58-62 (2014)

4. Gardner, W. Nonstationary learning characteristics of the LMS algorithm[J]. IEEE Transactions on Circuits and Systems, 34(10), 1199-1207 (2003)

5. Ye, F., H. Tian, and F. Che. CW interference effects on the performance of GPS receivers[C]. 2017 Progress In Electromagnetics Research Symposium - Fall (PIERS FALL), 66-72 (2017)

6. Betz J W, Kolodziejski K R . Generalized Theory of Code Tracking with an Early-Late Discriminator Part II: Noncoherent Processing and Numerical Results[J]. IEEE Transactions on Aerospace \& Electronic Systems, 45(4), 1538-1556 (2009)

7. Li Yawei. Research on Jamming and Anti-jamming about Satellite Navigation Receiver with Array Antenna[D]. Xidian University (2017)

8. Liu F, Wang Y, Yue L, et al. JAMMING METHOD BASED ON OPTIMAL POWER DIFFERENCE FOR LMS-GPS RECEIVER[J]. Progress in Electromagnetics Research M, 77, 167-175 (2019)

9. Liu F, Du R , Bai X . A virtual space-time adaptive beamforming method for spacetime antijamming[J]. Progress in Electromagnetics Research M, 58, 183-191 (2017)

10. Turan C. A new variable step-size block LMS algorithm for a non-stationary sparse systems[C] Twelve International Conference on Electronics Computer \& Computation. $\operatorname{IEEE}(2016)$ 